\title{
Signal Recovery With Cost-Constrained Measurements
}

\author{
Ayça Özçelikkale, Member, IEEE, Haldun M. Ozaktas, Member, IEEE, and Erdal Arıkan, Senior Member, IEEE
}

\begin{abstract}
We are concerned with the problem of optimally measuring an accessible signal under a total cost constraint, in order to estimate a signal which is not directly accessible. An important aspect of our formulation is the inclusion of a measurement device model where each device has a cost depending on the number of amplitude levels that the device can reliably distinguish. We also assume that there is a cost budget so that it is not possible to make a high amplitude resolution measurement at every point. We investigate the optimal allocation of cost budget to the measurement devices so as to minimize estimation error. This problem differs from standard estimation problems in that we are allowed to "design" the number and noise levels of the measurement devices subject to the cost constraint. Our main results are presented in the form of tradeoff curves between the estimation error and the cost budget. Although our primary motivation and numerical examples come from wave propagation problems, our formulation is also valid for other measurement problems with similar budget limitations where the observed variables are related to the unknown variables through a linear relation. We discuss the effects of signal-to-noise ratio, distance of propagation, and the degree of coherence (correlation) of the waves on these tradeoffs and the optimum cost allocation. Our conclusions not only yield practical strategies for designing optimal measurement systems under cost constraints, but also provide insights into measurement aspects of certain inverse problems.
\end{abstract}

Index Terms-Distributed estimation, error-cost tradeoff, experiment design, fractional Fourier transform, measurement design, random field estimation, rate distortion, sensing, wave propagation.

\section{INTRODUCTION}

$\mathbf{T}$ HE problem addressed in this work was motivated mostly by problems related to measurement of propagating wavefields. We are concerned with the problem of estimating the values of a wave-field in a certain region from measurements of its values at another region. How well we can do this has to do with how much information the measured values carry about the unknown values. A study of these issues can also lead to a better understanding of what happens to the information carried by a wave-field as it propagates. Utilization of signal processing and information theory concepts in wave propagation problems has a long history, which we can here provide only a limited sample of. The concept of number of degrees of freedom

Manuscript received August 07, 2009; accepted February 16, 2010. Date of publication March 22, 2010; date of current version June 16, 2010. The associate editor coordinating the review of this manuscript and approving it for publication was Dr. Soontorn Oraintara. The work of A. Özçelikkale was supported by TÜBITAK Doctoral Scholarship. The work of H. M. Ozaktas was supported in part by the Turkish Academy of Sciences.

The authors are with the Department of Electrical Engineering, Bilkent University, TR-06800, Ankara, Turkey (e-mail: ayca@ee.bilkent.edu.tr; haldun@ee.bilkent.edu.tr; arikan@ee.bilkent.edu.tr).

Digital Object Identifier 10.1109/TSP.2010.2046435 is used in several works including [1]-[11]. Other works have adopted a sampling theory approach [12], [13]. The concepts of structural and metrical information discussed in [14] have found application in [2], [15], and [16]. A number of works utilizing information theoretic concepts such entropy or channel capacity in different contexts have appeared [17]-[25]. Nevertheless, we are not aware of any works which try to address wave measurement problems of the kind dealt with in this paper.

The linear wave equation is of fundamental importance in many areas of science and engineering. It governs the propagation of electromagnetic, optical, acoustic, and other kinds of fields so that the problem we discuss is of interest in a wide variety of contexts. The solution of the wave equation can be expressed in many forms. One of these is to express the field over one region as a linear superposition integral over another region.

In this paper, we consider a very general measurement scenario. Let us consider a wave-field propagating through a system characterized by a linear input-output relationship. We wish to recover the input wave field as economically as possible from noisy measurements of the output field. We are concerned with accuracy both in the sense of spatial resolution and in the sense of the amplitude resolution. We are also concerned with the cost of performing the measurements and the tradeoffs between the total cost and estimation accuracy. The cost of a measurement device is primarily determined by the number of amplitude levels that the device can reliably distinguish; devices with higher numbers of distinguishable levels have higher costs. We also assume that there is a limited cost budget so that it is not possible to make a high amplitude resolution measurement at every point. For a given cost budget, we would like to know how to best make the measurements so as to minimize the estimation error, or vice versa, leading to a tradeoff. In particular, we are interested in questions such as how many measurements we should make, how the sensitivity of each detector should be chosen, and so forth, in order to obtain the best tradeoff. These questions are not merely of interest for practical purposes but can also lead to a better understanding of the information relationships inherent in propagating wave-fields.

While our primary motivation and numerical examples come from wave propagation problems, we emphasize that our formulation is also valid for other measurement problems where similar cost-budget models are applicable, and the observed variables are related to the unknown variables through a linear relation. One such example is the Wiener filtering problem which is a basic problem in signal processing with many practical applications. Another example arises in data communications, where a transmitted signal may suffer intersymbol interference as it passes through a medium, and the equalization problem is to estimate the transmitted signal from the received signal. These 
problems are of the same general structure as the one we are considering. In digital implemention of such estimators, the usual approach is to work with constant accuracy over all samples of the observation. Our framework introduces great flexibility in terms of the number, positions, and accuracies of these samples. This not only allows better optimization, but also allows us to observe a number of interesting tradeoffs and relationships.

In this paper, we formulate and solve a measurement strategy problem which arises in a diversity of physical contexts. We are concerned with measurement and estimation of spatially (or temporally) distributed fields modeled as random vectors. An important aspect of our formulation is that it allows sensors with different performances and costs in the model. While the kind of cost function we use may come as natural in the context of communication costs, we believe it has never been used to model the cost of measurement devices. The optimal measurement problem we define differs from standard estimation problems in that we are allowed to "design" the number and noise levels of the measurement devices subject to a total cost constraint. Our main results are presented in the form of tradeoff curves between the estimation error and the total cost. We discuss the effects of signal-to-noise ratio (SNR), distance of propagation, and the degree of coherence on these tradeoffs in wave-propagation problems. The degree of coherence is a measure of the amount of correlation among different points of a random wave-field. We are not aware of previous discussion of the effects of distance of propagation and degree of coherence in these types of problems. Our conclusions not only yield practical strategies for designing optimal measurement systems under cost constraints, but also provide interesting insights into the nature of information transfer in wave propagation.

In Section II of the paper, we present the mathematical model of the measurement problem discussed above. A fundamental concept in our formulation, the cost of a measurement, is discussed in Section III. Some special cases of our formulation are presented in Section IV. In Section V, we propose an iterative algorithm and provide numerical results. We conclude in Section VI.

\section{PROBLEM FORMULATION}

In the specific measurement scenario under consideration in this paper, noisy measurements are made at the output of a linear system, in order to estimate the input of the system. We study a discrete version of this problem by assuming that the space variable is discretized to a fixed finite set of points. The following development was first proposed in [26]-[28].

The system we consider may be represented by a matrix equation

$$
\mathbf{g}=\mathbf{H f}+\mathbf{n}
$$

where $\mathbf{f} \in \mathbb{R}^{N}$ is the unknown input random vector, $\mathbf{n} \in \mathbb{R}^{M}$ is the random vector denoting the inherent system noise, and $\mathrm{g} \in \mathbb{R}^{M}$ is the output of the linear system. We assume that $\mathbf{f}$ and $\mathbf{n}$ are statistically independent zero-mean random vectors. Here $\mathbf{H}$ is a $M \times N$ matrix denoting the linear system. We put no restrictions on the system matrix $\mathbf{H}$. For instance, in wave propagation applications, the locations of the measurements and the locations of the unknown field values may be quite distant

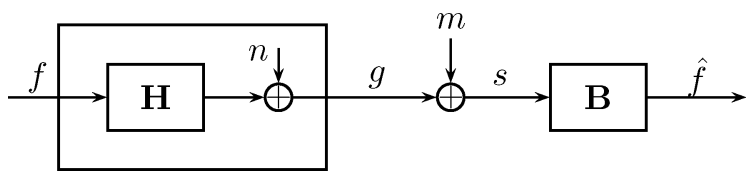

Fig. 1. Measurement and estimation systems model block diagram.

from each other, e.g., we may wish to estimate the field at the outer edges of a region with measurements made in the center.

Measurements are made at the output of the linear system to obtain the measurement vector $\mathbf{s} \in \mathbb{R}^{M}$ according to the measurement model

$$
\mathbf{s}=\mathrm{g}+\mathbf{m}
$$

where $\mathbf{m}$ denotes the measurement noise. We assume that $\mathbf{m}$ is independent of $\mathbf{f}$ and $\mathbf{n}$. Further, we assume that the components of $\mathbf{m}$ are independent, zero-mean random variables, but not necessarily identically distributed. So, the variance $\sigma_{m_{i}}^{2}$ of each component of $\mathbf{m}$, indexed by $i=1, \ldots, M$, may be different. Here $\mathbf{n}$ is an intrinsic part of the relation between $\mathbf{g}$ and f which we have no control over, whereas $\mathbf{m}$ is the noise associated with the measurement devices we use and thus depends on the choices we make.

In the following formulation, we assume the knowledge of only second-order statistics of the underlying random variables. We let $\mathbf{K}_{\mathbf{f}}, \mathbf{K}_{\mathbf{n}}, \mathbf{K}_{\mathbf{m}}$, and $\mathbf{K}_{\mathbf{s}}$ denote the covariance matrices of $\mathbf{f}, \mathbf{n}, \mathbf{m}$, and $\mathbf{s}$, respectively. Note that $\mathbf{K}_{\mathbf{s}}=\mathbf{H} \mathbf{K}_{\mathbf{f}} \mathbf{H}^{\mathrm{T}}+\mathbf{K}_{\mathbf{n}}+$ $\mathbf{K}_{\mathbf{m}}$. Note also that since we assume that $\mathbf{m}$ has independent components, $\mathbf{K}_{\mathbf{m}}=\operatorname{diag}\left(\sigma_{m_{1}}^{2}, \ldots, \sigma_{m_{M}}^{2}\right)$.

We assume that the cost associated with measuring the $i$ th component of $\mathbf{g}$ is $C_{i}=(1 / 2) \log \left(\sigma_{s_{i}}^{2} / \sigma_{m_{i}}^{2}\right)$, where $\sigma_{s_{i}}^{2}$ denotes the variance of $s_{i}$. The units of $C_{i}$ are bits. Smaller measurement noise levels result in higher costs whereas larger measurement noise levels allow lower costs. The plausibility of this form for the cost function is discussed in Section III. The cost of measuring $\mathrm{g}$ is defined as $\sum_{i=1}^{M} C_{i}$, the sum of the costs of measuring all of its components.

The objective is to minimize the mean-square error (MSE) between $\mathbf{f}$ and $\hat{\mathbf{f}}(\mathbf{s})$, the estimate of $\mathbf{f}$ given $\mathbf{s}$. We consider only linear minimum mean-square error (LMMSE) estimators, and $\hat{\mathbf{f}}(\mathbf{s})$ denotes the LMMSE estimator. Hence, the estimate may be written as $\hat{\mathbf{f}}(\mathbf{s})=\mathbf{B} \mathbf{s}$ where $\mathbf{B}$ is an $N$ by $M$ matrix. A block diagram illustrating this problem is given in Fig. 1.

The problem can be stated as follows: Given the covariance matrices $\mathbf{K}_{\mathbf{f}} \in \mathbb{R}^{N \times N}, \mathbf{K}_{\mathbf{n}} \in \mathbb{R}^{\mathrm{M} \times \mathrm{M}}$, and a system matrix $\mathbf{H} \in \mathbb{R}^{M \times N}$, determine

$$
\begin{aligned}
\varepsilon\left(C_{\text {tot }}\right) & \triangleq \min _{\mathbf{K}_{\mathbf{m}}} E\left\{\|\mathbf{f}-\hat{\mathbf{f}}(\mathbf{s})\|^{2}\right\} \\
& =\min _{\mathbf{K}_{\mathbf{m}}} \min _{\mathbf{B}} E\left\{\operatorname{tr}\left[(\mathbf{f}-\mathbf{B s})(\mathbf{f}-\mathbf{B s})^{\mathrm{T}}\right]\right\}
\end{aligned}
$$

subject to

$$
\sum_{i=1}^{M} \frac{1}{2} \log \left(\frac{\sigma_{s_{i}}^{2}}{\sigma_{m_{i}}^{2}}\right) \leq C_{\text {tot }} .
$$

where $\mathbf{K}_{\mathbf{m}}=\operatorname{diag}\left(\sigma_{m_{1}}^{2}, \ldots, \sigma_{m_{M}}^{2}\right)$ is the covariance of $\mathbf{m}, E$ denotes expectation with respect to $\mathbf{f}, \mathbf{n}$, and $\mathbf{m},\|\cdot\|$ denotes Euclidean norm, and tr denotes the trace operator. $C_{\text {tot }}$ is the total cost budget; the sum of the cost of all detectors is not allowed to 
exceed $C_{\text {tot }}$. We go from (3) to (4) by writing $E\left\{\|\mathbf{f}-\hat{\mathbf{f}}(\mathbf{s})\|^{2}\right\}=$ $\left.E\{\| \mathbf{f}-\mathbf{B s}) \|^{2}\right\}=E\left\{\sum_{i=1}^{N}(\mathbf{f}-\mathbf{B s})_{i}^{2}\right\}=E\{\operatorname{tr}[(\mathbf{f}-\mathbf{B s}) \cdot$ $\left.\left.(\mathbf{f}-\mathbf{B s})^{\mathrm{T}}\right]\right\}$. A list of selected variables and parameters is given in Table I.

We note that for a given $\mathbf{K}_{\mathrm{m}}$, the minimization over $\mathbf{B}$ in (4) is a standard LMMSE problem with solution $\mathbf{B}=\mathbf{K}_{\mathbf{f}} \mathbf{H}^{\mathrm{T}} \mathbf{K}_{s}^{-1}$. This standard solution may be arrived at using the orthogonality condition $E\left\{(\mathbf{f}-\mathbf{B s}) \mathbf{s}^{\mathrm{T}}\right\}=\mathbf{0} \in \mathbb{R}^{N \times M}$, where $E\left\{\mathbf{f} \mathbf{s}^{\mathrm{T}}\right\}=$ $\mathbf{K}_{\mathbf{f}} \mathbf{H}^{\mathrm{T}}$. Hence, we obtain:

$$
\varepsilon\left(C_{\text {tot }}\right)=\min _{\mathbf{K}_{\mathbf{m}}} \operatorname{tr}\left(\mathbf{K}_{\mathbf{f}}-\mathbf{K}_{\mathbf{f}} \mathbf{H}^{\mathrm{T}} \mathbf{K}_{\mathbf{s}}^{-1} \mathbf{H} \mathbf{K}_{\mathbf{f}}\right)
$$

subject to (5). In other words, our aim is to minimize the estimation error by allocating a given measurement cost budget optimally over the $M$ components of (2). This is equivalent to optimally adjusting the measurement noise level for each component, realizing that with a given budget, we cannot measure all components as highly accurately as we wish. Although not explicitly stated, the number of components we actually measure is also an optimization variable. If as the result of our optimization we find that $C_{i} \approx 0$ for certain components, this means that measuring those components do not usefully contribute to the estimation and therefore need not be measured in the first place.

As seen above, this problem differs from a standard LMMSE estimation problem in that the covariance $\mathbf{K}_{\mathbf{m}}$ of the measurement noise is subject to optimization. We are allowed to "design" the noise levels of the measurement devices subject to a total cost constraint so as to minimize the overall estimation error. To the best of our knowledge this problem is novel.

Our formulation can be easily generalized to allow repeated measurements (more than one measurement of any $g_{i}$ is allowed); however repeated measurements always yield higher error for a given cost budget hence including them in the model does not provide a better performance. This point is discussed in Section IV-1).

\section{COST FunCtion}

What we refer to as a measurement device is an instrument which can measure the value of a scalar physical quantity over some range with some resolution in amplitude. The cost of a measurement device is primarily determined by the number of amplitude levels that the device can reliably distinguish, a notion which is sometimes referred to as its dynamic range (although the term is sometimes also used to refer to an interval). We will assume that the ranges of measurement devices can be chosen freely to match any interval, and that this has no effect on the cost of the measurements provided the number of resolvable levels remains the same (similar to scaling the range of a multimeter). For a given linear system, the ranges of the measurement devices depend only on the given covariances. Therefore, they need to be changed only if the covariances change. Given the variances of $g$ and $m$ in the measurement process $s=g+m$, the number of distinguishable levels can be quantified as

$$
\rho=\varrho \frac{\sigma_{s}}{\sigma_{m}}=\varrho \sqrt{\left(1+\frac{\sigma_{g}^{2}}{\sigma_{m}^{2}}\right)}
$$

where $\varrho>0$ is a constant that depends on how reliably the levels need to be distinguished. In using this expression we are
TABLE I

SELECTED VARIABLES AND PARAMETERS

\begin{tabular}{cl}
\hline $\mathbf{f} \in \mathbb{R}^{N}$ & unknown input \\
$\mathbf{H} \in \mathbb{R}^{M \times N}$ & linear system \\
$\mathbf{n} \in \mathbb{R}^{M}$ & inherent system noise \\
$\mathbf{g} \in \mathbb{R}^{M}$ & output of the linear system \\
$\mathbf{m} \in \mathbb{R}^{M}$ & measurement noise \\
$\mathbf{s} \in \mathbb{R}^{M}$ & result of the measurement \\
$\mathbf{B} \in \mathbb{R}^{N \times M}$ & linear estimator \\
$\mathbf{K}_{\mathbf{x}}$ & covariance of $\mathbf{x}$ \\
$C_{i}$ & cost of measuring $g_{i}$ \\
$C_{\text {tot }}$ & cost budget \\
$\alpha$ & degree of coherence parameter \\
SNR & ratio of signal power to \\
& inherent system noise power
\end{tabular}

following the same rationale used to define the number of distinguishable signal levels at the receiver of an additive noise channel, which is due to Hartley [29], and further discussed in [30] and [31]. The square-root in the expression keeps the number of levels invariant under scaling of the signals by any constant. Clearly, in the limit of very noisy measurements, $\varrho$ should be 1 ; therefore, we set $\varrho=1$ henceforth.

We now list some properties that any plausible cost function must possess.

1) $C(\rho)$ must be a nonnegative, monotonically increasing function of $\rho$, with $C(1)=0$ since a device with one measurement level gives no useful information.

2) For any integer $L \geq 1$, we must have $L C(\rho) \geq C\left(\rho^{L}\right)$. This is because a measurement device with $\rho$ levels can be used $L$ times in succession with range adjustments between measurements, to distinguish $\rho^{L}$ levels. (We also note that this property may be expressed in a more general form as $\sum_{i=1}^{L} C\left(\rho_{i}\right) \geq C\left(\prod_{i=1}^{L} \rho_{i}\right)$.)

A function possessing these properties is the logarithm function; therefore we propose

$$
C(\rho)=\log \rho=\frac{1}{2} \log \left(\frac{\sigma_{s}^{2}}{\sigma_{m}^{2}}\right)=\frac{1}{2} \log \left(1+\frac{\sigma_{g}^{2}}{\sigma_{m}^{2}}\right)
$$

as the cost of carrying out a measurement $s=g+m$.

The proposed cost function has the same form as Shannon's formula for the capacity of a Gaussian noise channel. This does not come as a surprise since a measurement process $s=g+m$ is analogous to sending a message $g$ across a communication channel that adds a noise term $m$ to it. On the other hand, the notion of adjusting the range while keeping the number of resolvable levels constant has no direct counterpart in the communication setting; hence, the measurement and communication problems are not identical problems. We believe such a cost function has never been used to model the cost of measurement devices.

In the communication problem, the amount of information delivered to the receiver is measured by the mutual information $I(s ; g)=h(s)-h(m)$ between the transmitted and re- 
ceived signals. $I(s ; g)$ is also an attractive candidate for the cost function in the measurement scenario since the value of a measurement would be quantified most fairly by how many bits of information it actually conveys on the average about the measured quantity. On the other hand, there is a practical difficulty in charging a fee $I(s ; g)$ as it depends on the actual probability distribution $p(g)$ of the measured quantity. It is logical that the measurement device manufacturer will try to sell its device at the price $\max _{p(g)} I(s ; g)$ where the maximum is calculated subject to a power constraint $E\left[g^{2}\right] \leq \sigma_{g}^{2}$. The would-be equipment purchaser on the other hand will not be willing to pay more than $\min _{p(m)} \max _{p(g)} I(s ; g)$ since she or he is only assured that $E\left[m^{2}\right] \leq \sigma_{m}^{2}$. Shannon [30] shows that this minmax problem is solved by the Gaussian densities for both $p(g)$ and $p(m)$ and the resulting minmax value is the expression $C(\rho)$ given in (8). Thus, the cost function we propose has a satisfying economic interpretation: the seller of the equipment assumes that the purchaser will make the best use of the equipment while the purchaser assumes that the equipment will give the worst type of measurement noise (which is Gaussian) for the given level of resolution.

Since Gaussian error is an acceptable model for many types of measurement devices, the cost function that we use makes sense in a wide range of contexts. For problems where measurement noise is known to follow a different distribution, the cost function can be modified accordingly.

Finally we explore the relationship of the measurement problem to rate-distortion theory. It is clear from Fig. 1 that, by the data-processing theorem [32], we have the following relationship regarding the mutual information of the related random vectors: $I(\mathbf{f} ; \hat{\mathbf{f}}) \leq I(\mathrm{~g} ; \mathbf{s})$; i.e., the estimate $\hat{\mathbf{f}}$ can only provide as much information about $\mathbf{f}$ as the measurement devices extract from the observable $\mathrm{g}$. In turn, by standard arguments, we have $I(\mathrm{~g} ; \mathbf{s}) \leq \sum_{i=1}^{M} I\left(g_{i} ; s_{i}\right)$. The cost function $(1 / 2) \log \left(1+\sigma_{g_{i}}^{2} / \sigma_{m_{i}}^{2}\right)$ that we use upper-bounds $I\left(g_{i} ; s_{i}\right)$ whenever the measurement noise is Gaussian with a given variance $\sigma_{m_{i}}^{2}$ and the variance of the measured quantity is fixed as $\sigma_{g_{i}}^{2}$. Thus, for Gaussian measurement noise, we have $I(\mathbf{f} ; \hat{\mathbf{f}}) \leq C_{\text {tot }}$ where $C_{\text {tot }}$ is the total measurement budget.

The goal of measurements is the minimization of the MSE $\varepsilon\left(C_{\text {tot }}\right)=E[d(\mathbf{f}, \hat{\mathbf{f}})]$ within a budget $C_{\text {tot }}$ where $d$ denotes $\|\mathbf{f}-\hat{\mathbf{f}}\|^{2}$. From a rate-distortion theory viewpoint, interpreting $d$ as a distortion measure, this is similar to minimizing the average distortion in the reconstruction of $\mathbf{f}$ from a representation $\hat{\mathbf{f}}$ subject to a rate constraint $I(\mathbf{f} ; \hat{\mathbf{f}}) \leq C_{\text {tot }}$. This viewpoint immediately gives the bound $\varepsilon\left(C_{\text {tot }}\right) \geq D\left(C_{\text {tot }}\right)$ where $D\left(C_{\text {tot }}\right)$ is the distortion-rate function applicable to this situation.

In the rate-distortion framework one is given complete freedom in forming the reconstruction vectors $\hat{\mathbf{f}}$ subject only to a rate constraint, which in measurement terminology would mean the ability to apply arbitrary transformations on the observable $\mathrm{g}$ before performing a measurement (so as to carry out the measurement in the most favorable coordinate system), and not being constrained to linear measurements or linear estimators. Thus, the measurement problem can be seen as a deviation from the rate-distortion problem in which the formation of the reconstruction vector is restricted by various constraints.

\section{SPECIAL CASES}

1) Repeated Measurements of the Field at a Single Point: As noted, repeated measurements of components of $\mathbf{g}$ are always suboptimal in the sense that doing so results in greater error for given cost. Here we allow more than one measurement of any component of $\mathbf{g}$ and show that this is indeed the case. We assume that different measurements are statistically independent conditional on $\mathrm{g}$ even if repeated measurements of the same component are in question.

First we consider the simple case in which repeated measurements are made at a single point $g_{i}$ and the other components of $\mathrm{g}$ are not measured. That is, one is allowed to make $P_{i}$ measurements on $g_{i}$ indexed by $j=1, \ldots, P_{i}$ as $s_{j}^{i}=g_{i}+m_{j}^{i}$ subject to the usual cost constraint. Here the subscript denotes the index of the component of $\mathbf{g}$ where the repeated measurements are made. Since no other component of $\mathbf{g}$ is measured, the total number of measurements is equal to the number of repeated components $P_{i}$, the measurement noise vector $\mathbf{m}^{i}=\left[m_{1}^{i}, \ldots, m_{P_{i}}^{i}\right]^{\mathrm{T}} \in$ $\mathbb{R}^{P_{i}}$, and the measurement vector $\mathbf{s}^{i}=\left[s_{1}^{i}, \ldots, s_{P_{i}}^{i}\right]^{\mathrm{T}} \in \mathbb{R}^{P_{i}}$. We consider the problem of estimation of a single component of the input field $f_{k}$ where $k \in 1, \ldots, N$. By studying this case, we wish to see which measurement strategy is better: i) to make one high quality measurement by renting the best device within budget limits, or ii) to split the budget among multiple lower quality devices. Simple LMMSE analysis shows that the first alternative is better, as we now show.

For any given allocation of noise variances $\left(\sigma_{m_{1}}^{2}, \ldots, \sigma_{m_{P_{i}}}^{2}\right)$, the $P_{i}$ measurements yield the LMMSE estimate $\hat{f}_{k}(\mathbf{s})=\mathbf{a}^{\mathrm{T}} \mathbf{s}$ where $\mathbf{a} \in \mathcal{R}^{P_{i}}$. Here, the components of $\mathbf{a}$ are obtained by solving the orthogonality conditions:

$$
a_{j}=\frac{E\left[f_{k} g_{i}\right]}{\sigma_{\mathrm{eq}}^{2}+\sigma_{g_{i}}^{2}} \frac{\sigma_{\mathrm{eq}}^{2}}{\sigma_{m_{j}}^{2}}
$$

where $\sigma_{\text {eq }}^{2}=\left(\sum_{j=1}^{P_{i}}(1) /\left(\sigma_{m_{j}}^{2}\right)\right)^{-1}$. The associated MSE is

$$
\varepsilon_{i}=\sigma_{f_{k}}^{2}-\frac{E\left[f_{k} g_{i}\right]^{2}}{\sigma_{\mathrm{eq}}^{2}+\sigma_{g_{i}}^{2}} .
$$

The total measurement cost for this scheme is $\sum_{j=1}^{P_{i}}(1 / 2) \log \left(1+\sigma_{g_{i}}^{2} / \sigma_{m_{j}}^{2}\right)$. We observe that among all schemes of allocation of noise variances yielding the same $\sigma_{\text {eq }}^{2}$ (hence giving the same MSE), the cost is minimized by taking $\sigma_{m_{j}}^{2}=\sigma_{\text {eq }}^{2}$ for any one of the indexes $j$ and $\sigma_{m_{j}}^{2}=\infty$ for the others. This corresponds to making one high quality measurement. Therefore, for a given error, the total cost is minimized by making one high quality measurement rather than many low quality ones. The error is a strictly decreasing function of the cost so that we can further conclude that this is also the strategy minimizing error for given cost.

We note that this result trivially holds when one wants to estimate the whole field vector $\mathbf{f} \in \mathbb{R}^{N}$ instead of a single component $f_{k}$ of the vector. It also remains true when other components of $\mathbf{g}$ are measured alongside with $g_{i}$, as can be shown by noting that the estimation errors for the components of $\mathrm{g}$ do not change as long as $\sigma_{\text {eq }}^{2}$ is the same, so that the estimator coefficients associated with these components and therefore the estimation error for $\mathbf{f}$ also do not change. Therefore, we conclude 
that allowing repeated measurements of the same point does not provide an opportunity for further optimization, since for every measurement scheme involving more than one measurement of the same component, it is certain that there is another scheme that yields the same error with a lower cost budget.

2) Diagonal Case: In order to see the relationship of our formulation with the "water-filling" solutions common in certain information-theoretic problems (e.g., [32, Ch. 9] and [33, Ch. 13]), we consider the special case where the matrix $N=M$, the matrix $\mathbf{K}_{\mathbf{f}}$ is diagonal, $\mathbf{K}_{\mathbf{n}}=\mathbf{0}$, and $H$ is the identity matrix.

In this special case, we have $N$ separate LMMSE problems tied together by a total cost constraint. By standard techniques [32, Ch. 9], [33, Ch. 13], we obtain the optimal detector variances that minimize the estimation error as

$$
\sigma_{m_{i}}^{2}= \begin{cases}\frac{\nu \sigma_{f_{i}}^{2}}{\sigma_{f_{i}}^{2}-\nu}, & \text { if } \sigma_{f_{i}}^{2}-\nu>0 \\ \infty, & \text { if } \sigma_{f_{i}}^{2}-\nu \leq 0\end{cases}
$$

where the parameter $\nu$ is selected so that the total cost is $C_{\text {tot }}$. Notice that for those components for which there is a nontrivial measurement $\left(\sigma_{m_{i}}^{2}<\infty\right)$, we have $1 / \sigma_{m_{i}}^{2}+1 / \sigma_{f_{i}}^{2}=1 / \nu$, which is reminiscent of the "water-filling" solutions referred to above.

3) Accurate Measurements (High Budget) Case: When the uncertainty introduced by the measurements are small with respect to the range of $g$, we refer to this case as the accurate measurements case. This is the case where $\mathbf{K}_{\mathrm{s}}$ is near $\mathbf{K}_{\mathrm{g}}$, where $\mathbf{K}_{\mathbf{g}}=\mathbf{H} \mathbf{K}_{\mathbf{f}} \mathbf{H}^{\mathrm{T}}+\mathbf{K}_{\mathbf{n}}$ is the covariance of $\mathbf{g}$. Hence, we may use the first order approximation of the inverse of a positive definite symmetric matrix to write $\mathbf{K}_{\mathrm{s}}^{-1} \approx \mathbf{K}_{\mathrm{g}}^{-1}-\mathbf{K}_{\mathrm{g}}^{-1} \mathbf{K}_{\mathrm{m}} \mathbf{K}_{\mathrm{g}}^{-1}$, and using the linearity of the trace operator, the MMSE can be written as

$\operatorname{tr}\left(\mathbf{K}_{\mathbf{f}}-\mathbf{K}_{\mathbf{f}} \mathbf{H}^{\mathrm{T}} \mathbf{K}_{\mathbf{g}}^{-1} \mathbf{H} \mathbf{K}_{\mathbf{f}}\right)+\operatorname{tr}\left(\mathbf{K}_{\mathbf{f}} \mathbf{H}^{\mathrm{T}} \mathbf{K}_{\mathbf{g}}^{-1} \mathbf{K}_{\mathbf{m}} \mathbf{K}_{\mathbf{g}}^{-1} \mathbf{H} \mathbf{K}_{\mathbf{f}}\right)$.

The error is expressed as the sum of two parts. The first part is independent of the accuracy of the measurements. For physical phenomena represented by noninvertible matrices $\mathbf{H}$, this irreducible error remains even if the measurements are perfect, and corresponds to the limited information transfer capability of the physical system. The second additive error component is due to the imperfect measurements. In this case the estimation error is a linear function of $\mathbf{K}_{\mathrm{m}}$, and the resulting optimization problem is convex. Since the objective and constraint functions are differentiable and Slater's condition holds, the Karush-Kuhn-Tucker (KKT) conditions are necessary and sufficient for optimality [34, Ch. 5]. Hence, by solving the KKT conditions, the optimal noise levels can be obtained as

$$
\sigma_{m_{i}}^{2}=\frac{-\sigma_{g_{i}}^{2}+\sqrt{\sigma_{g_{i}}^{4}+\frac{4 \sigma_{g_{i}}^{2}}{\nu d_{i i}}}}{2}
$$

where $\nu>0$ is a parameter chosen so that the total cost is $C_{\text {tot }}$, and $d_{i i}$ 's are the diagonal elements of $\mathbf{D}=\mathbf{K}_{\mathbf{g}}^{-1} \mathbf{H} \mathbf{K}_{\mathbf{f}}^{2} \mathbf{H}^{\mathrm{T}} \mathbf{K}_{\mathbf{g}}^{-1}$.

\section{NUMERICAL RESULTS}

First, we present the algorithm we employed for solving the optimization problem (6). Our algorithm is based on (4) and relies on taking turns in fixing $\mathbf{B}$ and $\mathbf{K}_{\mathbf{m}}$ and minimizing over

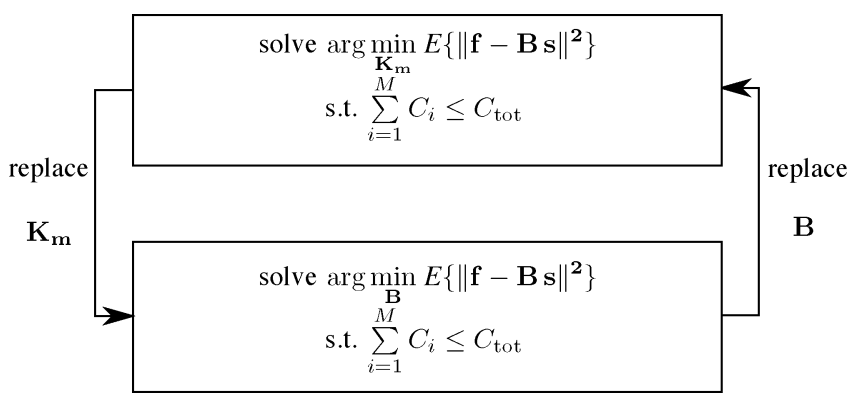

Fig. 2. Block diagram of the algorithm.

the other. For fixed $\mathbf{K}_{\mathbf{m}}$, the optimal value of the linear estimator $\mathbf{B}$ that minimizes the error can be analytically written in terms of $\mathbf{K}_{\mathrm{m}}$ as $\mathbf{B}=\mathbf{K}_{\mathbf{f}} \mathbf{H}^{\mathrm{T}}\left(\mathbf{H} \mathbf{K}_{\mathbf{f}} \mathbf{H}^{\mathrm{T}}+\mathbf{K}_{\mathbf{n}}+\mathbf{K}_{\mathrm{m}}\right)^{-1}$. On the other hand, if we fix $\mathbf{B}$, the problem is to minimize $\operatorname{tr}\left(\mathbf{B K}_{\mathbf{m}} \mathbf{B}^{\mathrm{T}}\right)$ over $\mathbf{K}_{\mathrm{m}}$ subject to (5). Since the differentiability and Slater's condition hold in this case as well, the optimal noise levels can be found as

$$
\sigma_{m_{i}}^{2}=\frac{-\sigma_{g_{i}}^{2}+\sqrt{\sigma_{g_{i}}^{4}+\frac{4 \sigma_{g_{i}}^{2}}{\eta p_{i i}}}}{2}
$$

by solving the KKT conditions. Here $\eta>0$ is a parameter chosen so that the total cost is $C_{\text {tot }}$, and $p_{i i}$ 's are the diagonal elements of $\mathbf{P}=\mathbf{B}^{\mathrm{T}} \mathbf{B}$.

The resulting algorithm is summarized as follows (Fig. 2): We initialize the algorithm by setting $t=0$ and $\mathbf{K}_{\mathbf{m}}^{(t=0)}$ to a random positive-definite diagonal matrix. At each iteration $t$, first we fix $\mathbf{K}_{\mathbf{m}}$ and set $\mathbf{B}^{(t+1)}=\mathbf{K}_{\mathbf{f}} \mathbf{H}^{\mathrm{T}}\left(\mathbf{K}_{s}^{(t)}\right)^{-1}$ where $\mathbf{K}_{s}^{(t)}=\mathbf{H K}_{\mathbf{f}} \mathbf{H}^{\mathrm{T}}+\mathbf{K}_{\mathbf{n}}+\mathbf{K}_{\mathbf{m}}^{(t)}$, which is the optimum value of $\mathbf{B}$ for $\mathbf{K}_{\mathrm{m}}^{(t)}$. Then we fix $\mathbf{B}$ and minimize over $\mathbf{K}_{\mathbf{m}}$ : We obtain $\mathbf{K}_{\mathbf{m}}^{(t+1)}$ by solving (14) with $p_{i i}$ replaced with $a_{i}=$ $\sum_{j=1}^{M}\left(b_{j, i}^{(t+1)}\right)^{2}$. For the stopping criterion, we use the relative error: if $\varepsilon\left(C_{\text {tot }}\right) / \operatorname{tr}\left(\mathbf{K}_{\mathbf{f}}\right)$ does not change by more than $10^{-4}$ over 10 consecutive iterations, we stop; otherwise, we increment $t$ and continue. The algorithm converges typically within $10-150$ iterations depending on the problem parameters. Details on this type of algorithm may be found in [35, Ch. 9].

The problem we formulate and solve in this paper was motivated by the physical problem of measuring propagating wave fields at a certain number of points and estimating the values of the field, possibly at other, distant locations. Although our formulation can handle very general cases of this problem, in our numerical examples we will focus on the case where there are two planar or spherical reference surfaces, perpendicular to the axis of propagation and separated by a certain distance. We assume that all measurement probes are placed uniformly on one surface and we desire to estimate the field on the other surface. In this case the measured field is related to the unknown field through a diffraction integral, a convenient approximation of which is the Fresnel diffraction integral or more generally a quadratic-phase integral (linear canonical transform) [36, Ch. 8], [37, Ch. 2], and [38], [39]. It is well known that these integrals can be expressed in terms of the fractional Fourier transform (FRT), which provides an elegant and pure description of these systems [40, Ch. 9], [41], and which has found many applications in signal processing [42]-[49]. The FRT is the fractional operator power of the Fourier transform with fractional 
order $a$. When $a=0$ the FRT reduces to the identity operation and when $a=1$ it reduces to the ordinary Fourier transform. Moreover, the transform is index-additive: the $a_{1}$ th transform of the $a_{2}$ th transform is equal to the $a_{1}+a_{2}$ th transform. Further information on the FRT and its computation may be found in [40] and [50]. Essentially, the FRT captures the underlying physics of wave propagation and diffraction phenomena in its purest form and is therefore suitable for modeling wave propagation for our present purposes. Thus, in our examples we will take the system matrix $\mathbf{H}$ to be the $N$ by $N$ real equivalent of the $N / 2$ by $N / 2$ complex FRT matrix. For the generation of FRT matrices of different orders, an implementation of the algorithm presented in [51] and in [40, Ch. 6] is used; this implementation is available at [52].

Propagating wave-fields may have different degrees of what is known as coherence. Highly coherent fields are those whose values at different points are highly correlated with each other. Highly incoherent fields are those whose values at different points are highly uncorrelated. Since we have observed that our results depend on the degree of coherence of the fields, we will consider several covariance matrices corresponding to different degrees of coherence (correlation between their components). It is known that highly coherent fields have covariance matrices whose eigenvalues are highly unevenly distributed. On the other hand, highly incoherent fields have eigenvalues which are nearly equal to each other [53]. To obtain covariance matrices with different degrees of coherence, we will choose the eigenvalues to be normally distributed with standard deviation equal to $N / \alpha$ pixels. Here the parameter $\alpha$ can also be interpreted as the number of standard deviations of the Gaussian covered by the $N$ samples. In our experiments $\alpha$ takes the values $\alpha=0.25,2,16,128,1024$, where $\alpha=1024$ corresponds to the case where all but one eigenvalue is negligible, and $\alpha=0.25$ corresponds to the case where all eigenvalues are nearly equal. While $\alpha$ is a convenient parameter to work with, we note that it should not be seen as a linear measure of the degree of coherence [53]. To generate the covariance matrices with these eigenvalues, we use the eigenvalue-eigenvector decomposition of a covariance matrix $\mathbf{K}=\mathbf{Q} \mathbf{\Lambda} \mathbf{Q}^{\mathrm{T}}$, where $\boldsymbol{\Lambda}=\operatorname{diag}\left(\varsigma_{i}\right)$. Here the orthogonal matrix $\mathbf{Q}$ is obtained by $\mathbf{Q R}$ decomposition of a $N \times N$ matrix with i.i.d. zero-mean Gaussian entries. For the system noise $\mathbf{n}$, the covariance matrix is generated similarly with $\alpha=4$ with a different $\mathbf{Q}$ matrix.

Another important parameter used in the experiments is

$$
\mathrm{SNR} \triangleq \frac{\operatorname{tr}\left(\mathbf{H} \mathbf{K}_{\mathbf{f}} \mathbf{H}^{\mathrm{T}}\right)}{\operatorname{tr}\left(\mathbf{K}_{\mathbf{n}}\right)}=\frac{\sum_{i=1}^{N} \sigma_{f_{i}}^{2}}{\sum_{i=1}^{M} \sigma_{n_{i}}^{2}}
$$

where the second form follows from $\mathbf{H}^{\mathrm{T}} \mathbf{H}=\mathbf{I}$ which in turn follows from the unitarity of the FRT. SNR measures the ratio of signal power to inherent system noise power, before measurements.

In the following experiments our main purpose will be to investigate the tradeoff between the MSE error $\varepsilon\left(C_{\text {tot }}\right)$ and measurement cost budget $C_{\text {tot }}$ after we have optimized over all possible allocations of cost over the measurement devices. The error will be reported as a percentage defined as $100 \varepsilon\left(C_{\text {tot }}\right) / \operatorname{tr}\left(\mathbf{K}_{\mathbf{f}}\right)$. The cost budget $C_{\text {tot }}$ is measured in bits by taking logarithms to base 2 . Unless otherwise stated all experiments are made with $a=0.5$ and $N=M=256$.

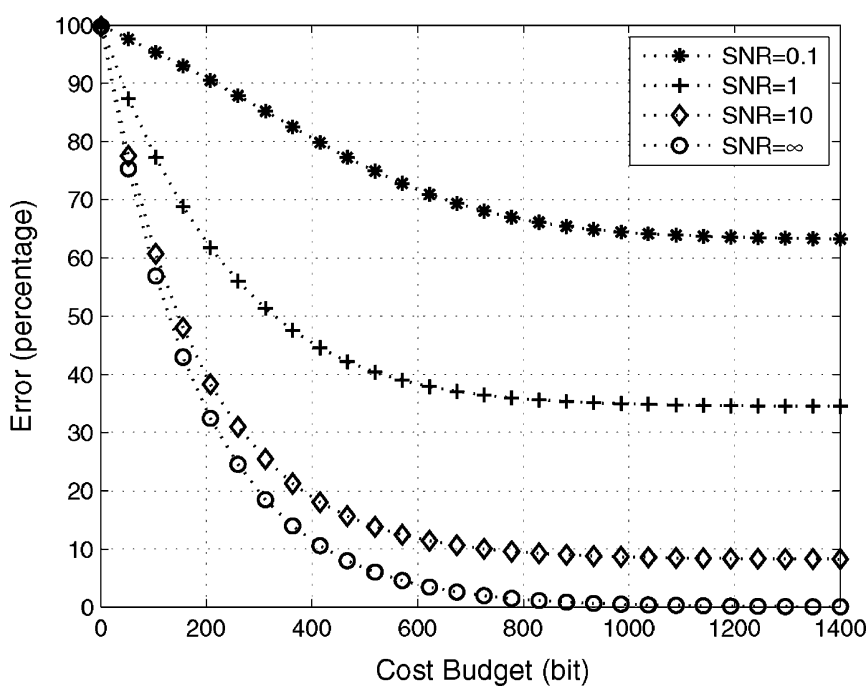

Fig. 3. Experiment 1: Error versus cost for $N=M=256, a=0.5, \alpha=$ 0.25, SNR variable.

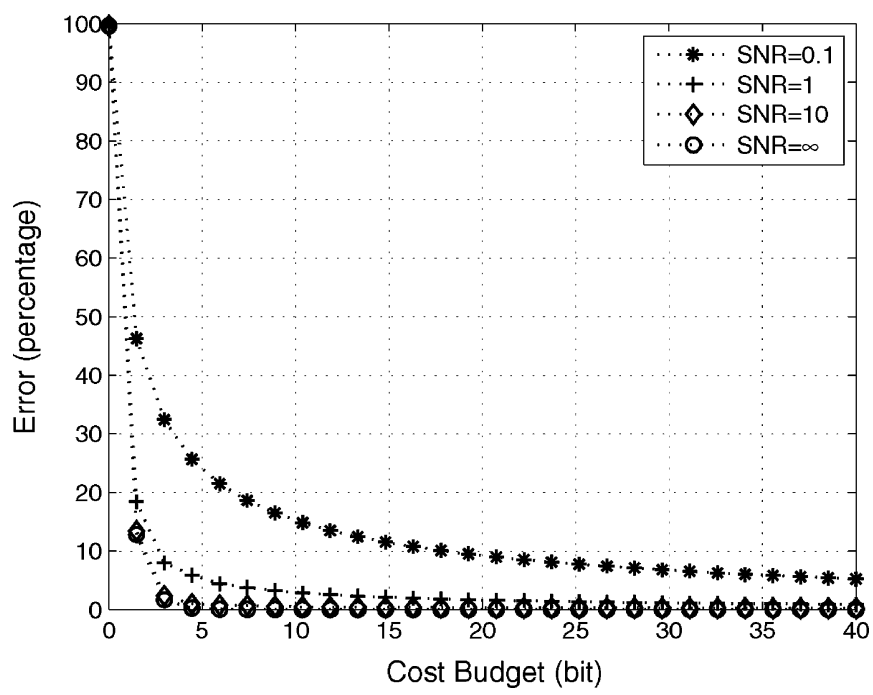

Fig. 4. Experiment 1: Error versus cost for $N=M=256, a=0.5, \alpha=$ 1024 , SNR variable.

Exp. 1: This experiment investigates the effect of SNR on the tradeoff between $C_{\text {tot }}$ and $\varepsilon\left(C_{\text {tot }}\right)$. In this experiment, SNR was variable, ranging over $0.1,1,10, \infty$ and two different values of $\alpha$ were considered. Figs. 3 and 4 give the curves for low and high $\alpha$ values, respectively. We notice that for both of the cases $\varepsilon\left(C_{\text {tot }}\right)$ is very sensitive to increases in $C_{\text {tot }}$ for smaller $C_{\text {tot }}$. Then it becomes less responsive and eventually saturates to the error value corresponding to zero measurement noise. For each value of cost, the error decreases as SNR increases, and for higher cost values will approach zero as SNR $\rightarrow \infty$. We see that when the field is more highly coherent (Fig. 4), we obtain much better tradeoff curves for all values of SNR than Fig. 3 which represents the highly incoherent extreme. For instance for $\mathrm{SNR}=\infty$, for the highly incoherent field an error of $10 \%$ is obtained at a cost of 400 bits, whereas for the highly coherent field the same error is achieved at a cost lower than 5 bits. This point is further investigated in Exp. 2. 


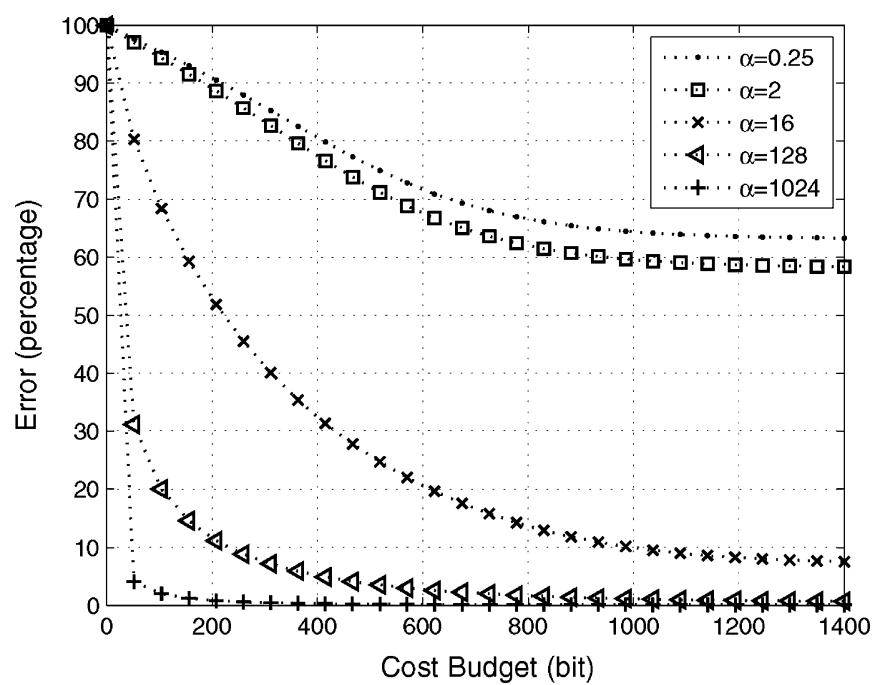

Fig. 5. Experiment 2: Error versus cost for $N=M=256, a=0.5, \mathrm{SNR}=$ $0.1, \alpha$ variable.

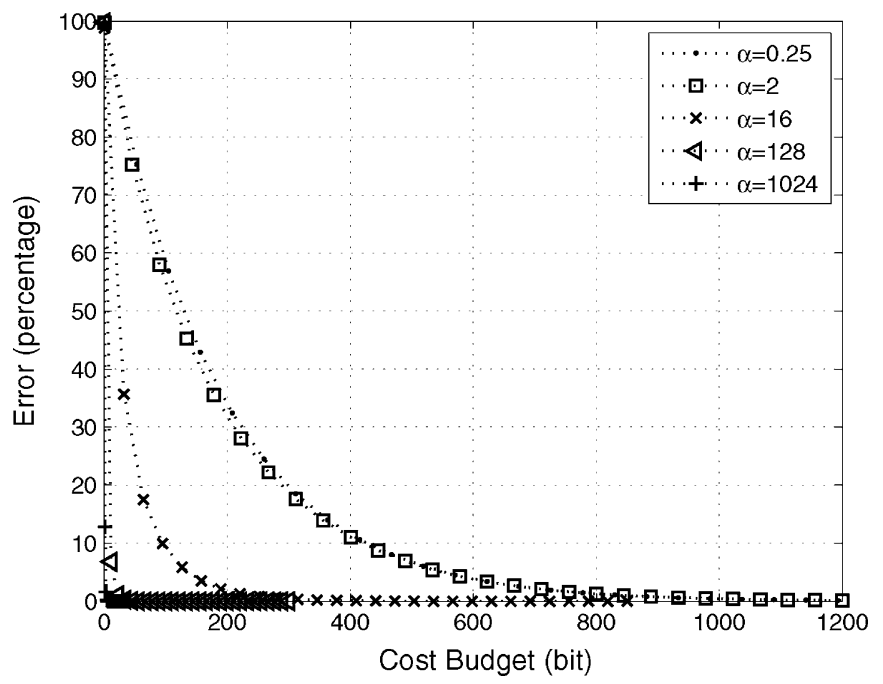

Fig. 6. Experiment 2: Error versus cost for $N=M=256, a=0.5$, SNR $=$ $\infty, \alpha$ variable.

Exp. 2: This experiment investigates the effect of degree of coherence of the unknown field on the tradeoff between $C_{\text {tot }}$ and $\varepsilon\left(C_{\text {tot }}\right)$. Figs. 5 and 6 show the results for two different SNR values $(\mathrm{SNR}=0.1$ and $\mathrm{SNR}=\infty$ ), for $\alpha=0.25,2,16,128,1024$. Both of the plots show that for low values of $\alpha$ corresponding to lower degrees of coherence, it is more difficult to achieve low values of error within a given budget. But as $\alpha$ increases, the total uncertainty in the field decreases, and it becomes a lot easier to achieve lower values of error. In fact, for high values of $\alpha$ and for low values of budget, the optimal strategy to minimize error turns out to be to measure the field value at only a few points with more accurate (and costly) measurement devices, rather than spreading the cost budget among many measurement points. This observation is further investigated in Exp. 4.

It is interesting to note that in all of the numerical examples we have considered, including the incoherent case, it is possible to reach with an average of 4 bits per component, the same error level that would be achieved with infinite accuracy (and cost).
Comparing the performances in Figs. 5 and 6 for low and high values of the cost budget, we see that for low budget values the effect of degree of coherence of the field can be considered more pronounced in the high SNR case, whereas for high budget values this effect is more pronounced in the low SNR case: For high values of cost budget, it is always possible to obtain very low values of error $(\approx 0)$ regardless of degree of coherence, when the SNR is high. But when the SNR is low and the cost budget is high, a substantial performance difference is observed between the correlated and uncorrelated fields, since it is possible to effectively cancel the effect of system noise $n$ when the degree of coherence of the field is high, yielding a better performance. When the budget is small and the SNR is low, although highly correlated fields lead to better performance, this improvement is limited by the presence of noise. When the budget is small but SNR is high, it is possible to obtain very low values of error $(\approx 0)$ when the field is highly correlated, resulting in a far better performance compared to the uncorrelated case.

Exp. 3: This experiment investigates the effect of SNR on the relationship between the number of effective measurements $M_{\text {eff }}$ and the budget $C_{\text {tot }}$. We will consider a measurement at a point to be effectively made if the cost of the measurement at this point is greater than $p\left(C_{\text {tot }} / N\right)$ bits. With this choice of threshold, it is guaranteed that the total cost of the measurements that are effectively made is higher than $(1-p) C_{\text {tot }}$. We use $p=0.125$. Measurements with less cost are very noisy measurements and do not contribute much either to the quality of the estimate or the total cost, so that it does not make much difference whether we actually perform them or not.

For $\alpha=0.25$, we see that one has to do measurements at all of the $M=256$ measurement points for all values of SNR and for all values of $C_{\text {tot }}$. This result is plausible since the field values are nearly uncorrelated in this case, and each point can be considered to provide new information.

The case of highly coherent fields is more interesting. Fig. 7 shows the results for $\alpha=1024$ with $\mathrm{SNR}=0.1,1,10, \infty$. For low values of SNR, the optimal strategy is to split the budget relatively broadly among the $M$ points. On the other hand, for high values of SNR, the best strategy is to allocate the budget to a smaller number of points. To understand this behavior, we observe that in this experiment the field values are highly correlated, hence the points measured carry nearly the same information. On the hand the system noise is highly uncorrelated. Based on these two observations, we can say that measuring a larger number of points increases the averaging effect and thus suppression of the system noise. Successively measuring highly correlated variables normally adds little information [so that one would prefer fewer but more accurate measurements instead]. However, when there is a lot of noise, the benefits of noise suppression can outweigh this so that a larger number of measurements are preferred.

Although the curves behave as if the number of effective measurements saturate at an asymptote for high values of cost budget, this is in fact not true and the number of effective measurements continue to increase as budget increases. This point is further discussed in the next experiment.

Exp. 4: This experiment investigates the effect of degree of coherence of the unknown field on the relationship between the number of effective measurements $M_{\text {eff }}$ and the budget $C_{\text {tot }}$. Fig. 8 shows the results for 


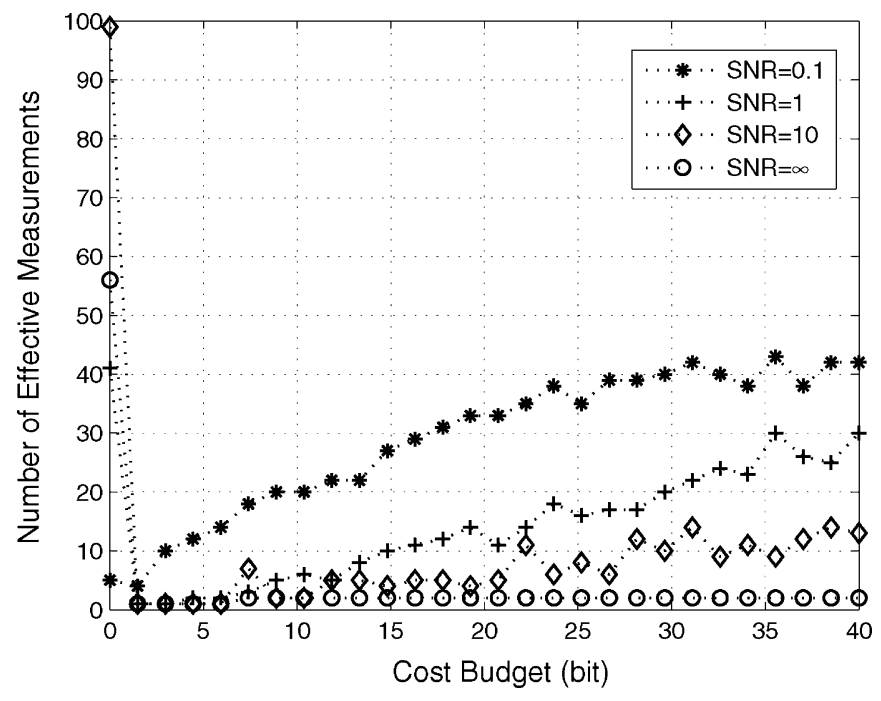

Fig. 7. Experiment 3: Effective number of measurements versus cost for $N=$ $M=256, a=0.5, \alpha=1024$, SNR variable.

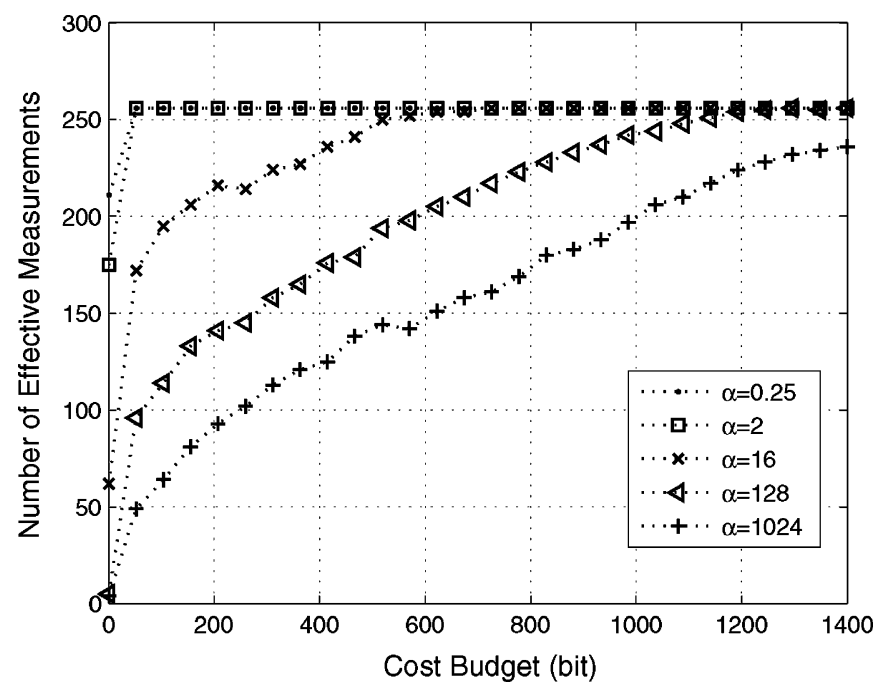

Fig. 8. Experiment 4: Effective number of measurements versus cost for $N=$ $M=256, a=0.5, \mathrm{SNR}=0.1, \alpha$ variable.

$\mathrm{SNR}=0.1, \alpha=0.25,2,16,128,1024$. We see that for all values of $\alpha$, and for low values of cost budget, the best strategy is to measure more accurately a relatively smaller number of points. But as the budget increases, the information that can be gained by measuring the field at a limited number of points with greater and greater accuracy saturates and splitting the budget over a larger number of measurement points become beneficial. For low values of $\alpha$, this shift in strategy takes place at lower values of cost budget. For a highly coherent field, the measurement of the field value at a particular point says much more about the field values at other points, and the benefit of measuring some of the field values with greater accuracy is prevailing.

Comparing this plot with Fig. 5 shows that the increase in the number of effective measurements for higher values of budget is not very meaningful since, for these budget values the error has almost reached its saturation value, but the algorithm being blind to this fact, increases the number of effective measurements to achieve tiny decreases in error. For instance, with $\alpha=$

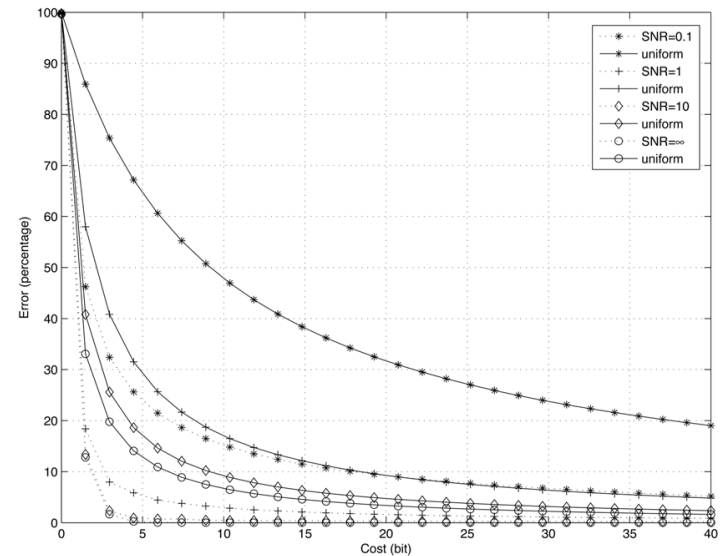

Fig. 9. Experiment 5: Error versus cost for $N=M=256, a=0.5, \alpha=$ 1024 , SNR variable. The dotted lines are for optimal cost allocation and the corresponding solid lines are for uniform cost allocation.

1024 , the error reaches a value of almost zero for a cost budget of 200 bits, and beyond this cost budget any increase in the number of measurements is made for the sake of a very small performance improvement.

We have also repeated the above experiment made for $\mathrm{SNR}=$ 0.1 for other values of SNR. We have observed that as SNR increases, a similar behavior is observed: the number of effective measurements again increases with increasing budget for all values of $\alpha$. But this time the rate of increase of the number of effective measurements with increasing budget is smaller. Also, at a given cost budget, the ratio of the number of effective measurements is larger for different values of $\alpha$. Hence, the difference in the optimum cost allocation strategies for different values of $\alpha$ is more apparent for higher values of SNR.

Exp. 5: This experiment aims to demonstrate how applying the optimum cost allocation strategy we have employed up to this point, improves the tradeoff between $C_{\text {tot }}$ and $\varepsilon\left(C_{\text {tot }}\right)$ compared to a simple uniform cost allocation strategy, where the cost budget is equally allocated: $C_{i}=C_{\text {tot }} / M, i=1, \ldots, M$. We expect that use of the optimal cost allocation will make a bigger difference for more highly coherent fields, since Exp. 4 shows that the optimum cost allocation is drastically different from a uniform cost allocation scheme. Furthermore, Exp. 3 suggests this effect should be more pronounced when SNR is high. Fig. 9 compares the tradeoff curves with optimum and uniform cost allocation schemes with $\alpha=1024, \mathrm{SNR}=0.1,1,10, \infty$. The dashed curves and the straight lines show the results for the optimum cost allocation scheme and the uniform cost allocation scheme respectively. As expected, for all values of SNR, the optimum cost allocation scheme gives significantly better tradeoffs compared to the uniform cost allocation case. For low $C_{\text {tot }}$ values, as SNR increases, the ratio of percentage error corresponding to uniform cost allocation to that corresponding to optimum cost allocation increases, showing that when the degree of coherence is high and the system noise is small, it is more important to optimize the allocation of the budget to the measurement points.

Exp. 6: This experiment investigates the effect of the fractional Fourier order on the tradeoff between $C_{\text {tot }}$ and $\varepsilon\left(C_{\text {tot }}\right)$. Fig. 10 presents the tradeoff curves with $\alpha=1024$, SNR $=0.1$, and $a=0,0.5,1$. The tradeoff curves obtained for different 


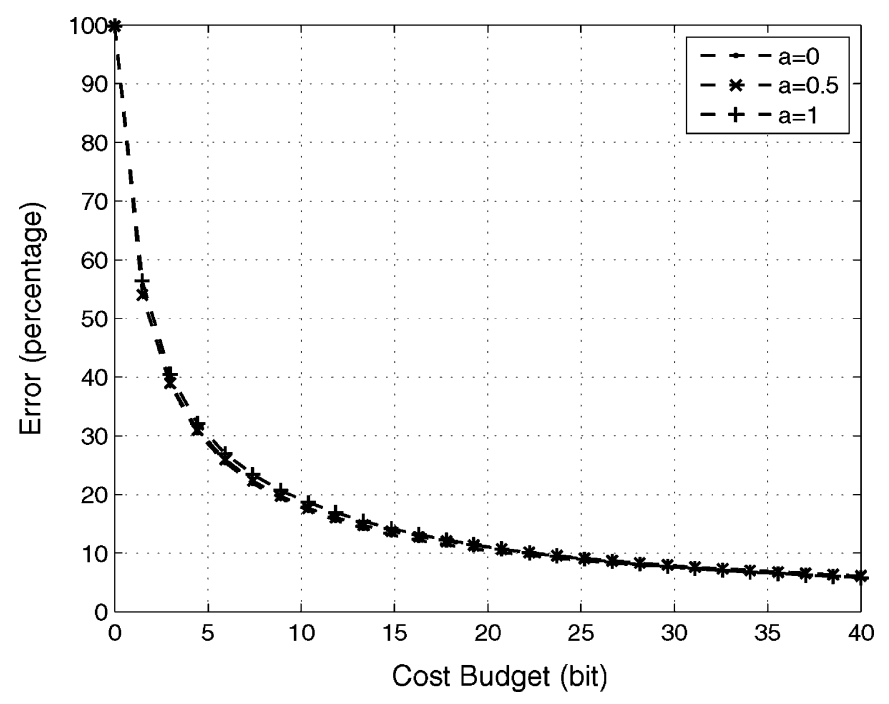

Fig. 10. Experiment 6: Error versus cost for $N=M=256, \mathrm{SNR}=$ $0.1, \alpha=1024, a$ variable.

values of $a$ are very close to each other, although higher values of $a$ yield slightly better curves. We have also repeated this experiment for different values of $\alpha$ and SNR. The resulting tradeoff curves in these other experiments exhibit even less difference for different FRT orders. Remembering that $a$ is a monotonic increasing function of the distance of propagation in wave propagation problems, these results show that the MSE is not critically dependent on how far the measurement devices are placed along the propagation axis.

Exp. 7: This experiment investigates the effect of making measurements at a smaller number of points. More specifically, we will examine the dependence of $\varepsilon\left(C_{\text {tot }}\right)$ on $M_{\mathrm{s}} \leq M$ for a fixed $C_{\text {tot }}$. Fig. 11 shows the results for $a=0.5, N=M=$ $256, \mathrm{SNR}=\infty, \alpha=16$ and $M_{\mathrm{s}}=8,16,32,64,128,256$. The measurement locations were chosen as uniformly spaced subgrids of the full 256-point grid (i.e., the grid for $M_{\mathrm{S}}=32$ was a sub-grid of that for $M_{\mathrm{s}}=64$ which was a sub-grid of that for $M_{\mathrm{s}}=128$, etc.). We see that for $M_{\mathrm{s}}=64$ and $M_{\mathrm{s}}=$ 128 roughly the same performance with the $M_{\mathrm{s}}=M=256$ case is observed, whereas for other values the performance degrades with decreasing $M_{\mathrm{s}}$. This behavior is related to the effective number of nonzero eigenvalues. For $\alpha=16$, the eigenvalues are samples of a Gaussian with standard deviation 256/16 pixels. Assuming the values of a Gaussian beyond its third standard deviation are negligible, the covariance matrix has about $3 \times 256 / 16=48$ nonzero eigenvalues. Indeed we observe that as long as the number of measurements $M_{\mathrm{s}}$ is higher than 48 , the tradeoff curves are similar to the $M_{\mathrm{s}}=M$ case. But if we restrict ourselves to do measurements at a smaller number of points such as $M_{\mathrm{S}}=8,16,32$, a substantial performance degradation is observed.

\section{CONCLUSION}

Motivated by problems related to measurement of propagating wave-fields, we formulated the problem of optimally measuring observed variables so as to estimate unknown variables under a total cost constraint. We proposed a measurement device model where each device has a cost depending on its resolving power. Based on this cost function we determine the

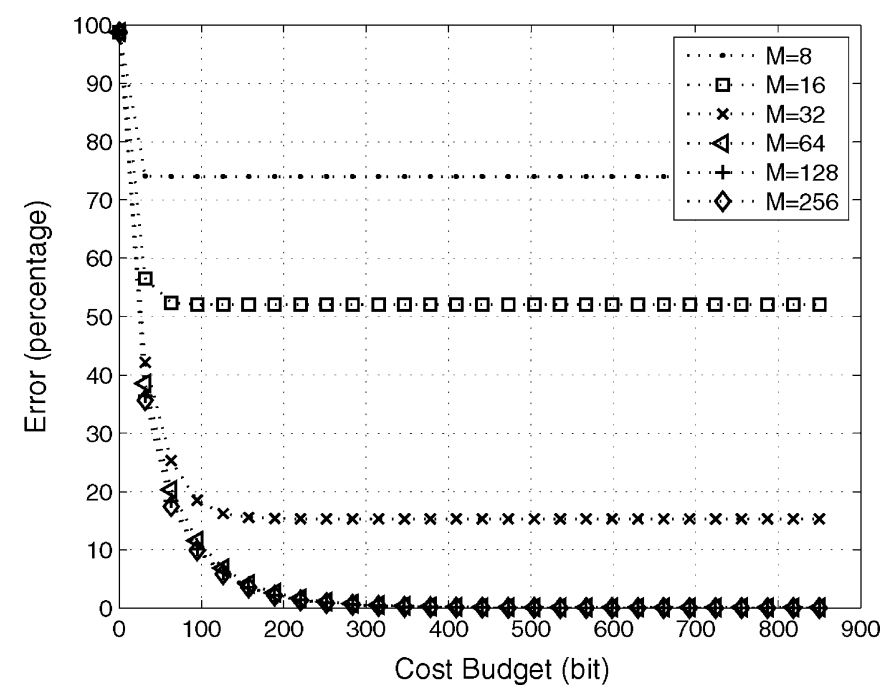

Fig. 11. Experiment 7: Error versus cost for $N=256, a=0.5, \alpha=$ $16, \mathrm{SNR}=\infty, M$ variable.

number of measurement devices and their accuracies that minimize estimation error for given total cost. We produce tradeoff curves between the error and the cost budget, corresponding to the optimal measurement strategy. We discuss the effects of SNR, distance of propagation, and the degree of coherence of the wave-fields on these tradeoffs.

Specific hardware may deviate from our hardware-independent cost-budget model to varying degrees. However, all measurement devices have finite accuracy and in general their cost is an increasing function of their accuracy. Therefore, we believe that the nature of the tradeoffs observed and the general conclusions and insights will remain useful under a wide variety of circumstances.

We have seen that making measurements with higher quality (and cost) measurement devices, should be preferred over making repeated measurements with lower cost (and quality) devices. This helps explain why it is better to make a limited number of high quality measurements when the field is highly coherent. At the other extreme of coherence, when the fields are uncorrelated, we noted that the best measurement strategy is a reverse-water filling scheme.

As expected, in our numerical experiments we observe that the estimation error decreases with increasing cost budget, and reaches zero error when there is no system noise. Not surprisingly, with increasing system noise levels (decreasing SNRs), poorer tradeoffs are observed. The cost-error tradeoff is greatly degraded by decreasing SNR for relatively incoherent fields, whereas it can be said to be less sensitive to SNR for coherent fields.

In general, it is possible to obtain better tradeoff curves for relatively coherent fields as compared to relatively incoherent fields for all values of SNR. The difference can be quite substantial and in the limit of full coherence/incoherence very large. For instance, for a coherent field, a total cost of a few bits may be sufficient to obtain a certain error, whereas for an incoherent field one may need a total cost which is of the order of $N$ times as large as this to achieve the same error. For relatively incoherent fields the best measurement strategy is to measure a greater number or most of the field components, whereas for relatively 
coherent light it is better to allocate the cost budget among a smaller number of field components. How small a number also depends on the SNR. It is preferable to measure a somewhat larger number of components when the SNR is low, but still many of the field components remain effectively unmeasured. These observations underline the fact that the degree of coherence (correlation) is a fundamental parameter that can have a significant effect on the results and therefore should be taken into consideration in order to ensure general applicability.

We also observed that in the wave propagation context the tradeoff curves are not significantly affected by how far from the unknown field the measurements are made. This allows us flexibility to accommodate practical constraints when choosing the measurement locations.

Finally we briefly discuss the relationship of the problem addressed in this paper with some earlier works which also involve estimation of desired quantities from measurements made from multiple sensors transmitting their observations to a decision center. The design of sensor and fusion center strategies has been studied in the context of different setups with different communication, computation, and power constraints [54]-[66]. A number of these works share some of the features of our formulation. In [56] and [59], problems related to wave propagation are studied with a statistical signal processing approach. Optimal sensor design has been studied in the form of quantizers or local encoders; for instance [54] and [55]. The problem of sensor selection as an estimation problem is considered in [63], and under given sensor performance and costs as a detection problem in [64]. The tradeoff between performance and total bit rate with a special emphasis on quantizer bit rates is studied in [57] and [58], where the estimation of a single parameter is considered. Although various aspects of the problem of sensing of physical fields have been widely studied as estimation problems, much of this work has loose connections with both the underlying physical phenomena and the physical aspects of the sensors employed. There seems to be a disciplinary boundary between these works and the works cited in Section I. Further work to bridge these two approaches will help us better understand the information theoretic relationships in physical fields and their measurement from a broader perspective.

\section{REFERENCES}

[1] G. T. Di Francia, "Resolving power and information," J. Opt. Soc. Amer., vol. 45, pp. 497-501, Jul. 1955.

[2] D. Gabor, "Light and information," in Progress in Optics, E. Wolf, Ed. Amsterdam, The Netherlands: Elsevier, 1961, vol. I, pp. 109-153, ch. 4.

[3] F. Gori and G. Guattari, "Shannon number and degrees of freedom of an image," Opt. Commun., vol. 7, pp. 163-165, Feb. 1973.

[4] A. Starikov, "Effective number of degrees of freedom of partially coherent sources," J. Opt. Soc. Amer., vol. 72, pp. 1538-1544, 1982.

[5] O. Bucci and G. Franceschetti, "On the degrees of freedom of scattered fields," IEEE Trans. Antennas Propag., vol. 37, pp. 918-926, Jul. 1989.

[6] D. Mendlovic and A. W. Lohmann, "Space-bandwidth product adaptation and its application to superresolution: Fundamentals," J. Opt. Soc. Amer. A, vol. 14, pp. 558-562, Mar. 1997.

[7] R. Piestun and D. A. B. Miller, "Electromagnetic degrees of freedom of an optical system," J. Opt. Soc. Amer. A, vol. 17, pp. 892-902, May 2000.

[8] A. Poon, R. Brodersen, and D. Tse, "Degrees of freedom in multipleantenna channels: A signal space approach," IEEE Trans. Inf. Theory, vol. 51, pp. 523-536, Feb. 2005.
[9] J. Xu and R. Janaswamy, "Electromagnetic degrees of freedom in 2-D scattering environments," IEEE Trans. Antennas Propag., vol. 54, pp. 3882-3894, Dec. 2006.

[10] M. Migliore, "On the role of the number of degrees of freedom of the field in MIMO channels," IEEE Trans. Antennas Propag., vol. 54, pp. 620-628, Feb. 2006.

[11] R. Kennedy, P. Sadeghi, T. Abhayapala, and H. Jones, "Intrinsic limits of dimensionality and richness in random multipath fields," IEEE Trans. Signal Process., vol. 55, no. 6, pp. 2542-2556, Jun. 2007.

[12] A. Stern and B. Javidi, "Analysis of practical sampling and reconstruction from Fresnel fields," Opt. Eng., vol. 43, pp. 239-250, Jan. 2004.

[13] L. Onural, "Exact analysis of the effects of sampling of the scalar diffraction field," J. Opt. Soc. Amer. A, vol. 24, pp. 359-367, Jan. 2007.

[14] D. MacKay, "Quantal aspects of scientific information," IEEE Trans. Inf. Theory, vol. 1, pp. 60-80, Feb. 1953.

[15] J. T. Winthrop, "Propagation of structural information in optical wave fields," J. Opt. Soc. Amer., vol. 61, pp. 15-30, Jan. 1971.

[16] T. W. Barret, "Structural information theory," J. Acoust. Soc. Amer., vol. 54, pp. 1092-1098, Oct. 1973.

[17] M. S. Hughes, "Analysis of digitized wave-forms using Shannon entropy," J. Acoust. Soc. Amer., vol. 93, pp. 892-906, Jan. 1993.

[18] D. Blacknell and C. J. Oliver, "Information-content of coherent images," J. Phys. D, vol. 26, pp. 1364-1370, Jan. 1993.

[19] F. T. Yu, Entropy and Information Optics. New York: Marcel Dekker, 2000.

[20] R. Konsbruck, E. Telatar, and M. Vetterli, "On the multiterminal rate-distortion function for acoustic sensing," in Proc. IEEE Int. Conf. Acoust., Speech Signal Process. (ICASSP), 2006, vol. 4, pp. 701-704.

[21] L. Hanlen and M. Fu, "Wireless communication systems with-spatial diversity: A volumetric model," IEEE Trans. Wireless Commun., vol. 5, pp. 133-142, Jan. 2006.

[22] M. Jensen and J. Wallace, "Capacity of the continuous-space electromagnetic channel," IEEE Trans. Antennas Propag., vol. 56, pp. 524-531, Feb. 2008.

[23] F. Gruber and E. Marengo, "New aspects of electromagnetic information theory for wireless and antenna systems," IEEE Trans. Antennas Propag., vol. 56, pp. 3470-3484, Nov. 2008.

[24] M. Migliore, "On electromagnetics and information theory," IEEE Trans. Antennas Propag., vol. 56, pp. 3188-3200, Oct. 2008.

[25] E. D. Micheli and G. A. Viano, "Inverse optical imaging viewed as a backward channel communication problem," J. Opt. Soc. Amer. A, vol. 26, pp. 1393-1402, Jan. 2009.

[26] A. Özçelikkale, "Structural and metrical information in linear systems," Master's thesis, Bilkent Univ., Ankara, Turkey, 2006.

[27] A. Özçelikkale, H. M. Özaktaş, and E. Arıkan, "Measurement strategies for input estimation in linear systems," in Proc. 2007 IEEE Signal Process. and Commun. App. Conf. (in Turkish) Transl.: Doğrusal sistemlerde girdi kestirimi İçin ölçüm yöntemleri, pp. 1-4.

[28] A. Özçelikkale, H. M. Ozaktas, and E. Arıkan, "Optimal measurement under cost constraints for estimation of propagating wave fields," in Proc. 2007 IEEE Int. Symp. Inf. Theory, pp. 696-700.

[29] R. V. L. Hartley, "Transmission of information," Bell Syst. Tech. J., vol. 7, pp. 535-563, Jul. 1928.

[30] C. E. Shannon, "A mathematical theory of communication," Bell Syst. Tech. J., vol. 27, pp. 379, 623-423, 656, Jul.-Oct. 1948.

[31] B. M. Oliver, J. R. Pierce, and C. E. Shannon, "The philosophy of PCM," in Proc. I.R.E., Nov. 1948, vol. 36, pp. 1324-1331.

[32] R. G. Gallager, Information Theory and Reliable Communication. New York: Wiley, 1968.

[33] T. M. Cover and J. A. Thomas, Elements of Information Theory. New York: Wiley, 1991

[34] S. Boyd and L. Vandenberghe, Convex Optimization. New York: Cambridge Univ. Press, 2004.

[35] J. Nocedal and S. J. Wright, Numerical Optimization. New York: Springer, 2006.

[36] P. M. Morse and K. U. Ingard, Theoretical Acoustics. Princeton, NJ: Princeton Univ. Press, 1986.

[37] C. A. Balanis, Antenna Theory: Analysis and Design. New York: Wiley, 2005.

[38] L. Onural and H. M. Ozaktas, "Signal processing issues in diffraction and holographic 3DTV," Signal Process.: Image Commun., vol. 22, pp. 169-177, Feb. 2007.

[39] M. J. Bastiaans, "Applications of the Wigner distribution function in optics," in The Wigner Distribution: Theory and Applications in Signal Processing, W. Mecklenbrauker and F. Hlawatsch, Eds. Amsterdam, The Netherlands: Elsevier, 1997, pp. 375-426. 
[40] H. M. Ozaktas, Z. Zalevsky, and M. A. Kutay, The Fractional Fourier Transform With Applications in Optics and Signal Processing. New York: Wiley, 2001.

[41] H. M. Ozaktas, B. Barshan, D. Mendlovic, and L. Onural, "Convolution, filtering, and multiplexing in fractional Fourier domains and their relation to chirp and wavelet transform," J. Opt. Soc. Amer. A, vol. 11, pp. 547-559, Feb. 1994

[42] M. A. Kutay, H. Özaktaş, H. M. Ozaktas, and O. Arıkan, "The fractional Fourier domain decomposition," Signal Process., vol. 77, pp. 105-109, Aug. 1999.

[43] M. F. Erden, M. A. Kutay, and H. M. Ozaktas, "Repeated filtering in consecutive fractional Fourier domains and its application to signal restoration," IEEE Trans. Signal Process., vol. 47, pp. 1458-1462, May 1999.

[44] H. M. Ozaktas and U. Sümbül, "Interpolating between periodicity and discreteness through the fractional Fourier transform," IEEE Trans. Signal Process., vol. 54, pp. 4233-4243, Nov. 2006.

[45] S. Qazi, A. Georgakis, L. K. Stergioulas, and M. Shikh-Bahaei, "Interference suppression in the Wigner distribution using fractional Fourier transformation and signal synthesis," IEEE Trans. Signal Process., vol. 55, no. 6, pp. 3150-3154, Jun. 2007.

[46] S.-C. Pei and J.-J. Ding, "Relations between Gabor transforms and fractional Fourier transforms and their applications for signal processing," IEEE Trans. Signal Process., vol. 55, no. 10, pp. 4839-4850, Oct. 2007.

[47] A. S. Amein and J. J. Soraghan, "Fractional chirp scaling algorithm: Mathematical model," IEEE Trans. Signal Process., vol. 55, no. 8, pp. 4162-4172, Aug. 2007.

[48] K. K. Sharma and S. D. Joshi, "Uncertainty principle for real signals in the linear canonical transform domains," IEEE Trans. Signal Process., vol. 56, no. 7, pp. 2677-2683, Jul. 2008.

[49] R. Tao, X.-M. Li, Y.-L. Li, and Y. Wang, "Time-delay estimation of chirp signals in the fractional Fourier domain," IEEE Trans. Signal Process., vol. 57, no. 7, pp. 2852-2855, Jul. 2009.

[50] A. Koç, H. M. Ozaktas, C. Candan, and M. A. Kutay, "Digital computation of linear canonical transforms," IEEE Trans. Signal Process., vol. 56, no. 6, pp. 2383-2394, Jun. 2008.

[51] Ç. Candan, M. A. Kutay, and H. M. Ozaktas, "The discrete fractional Fourier transform," IEEE Trans. Signal Process., vol. 48, no. 5, pp. 1329-1337, May 2000.

[52] Ç. Candan, "Discrete fractional Fourier transform matrix generator," [Online]. Available: http://www.ee.bilkent.edu.tr/ haldun/dFRT.m Jul. 1998

[53] H. M. Ozaktas, S. Yüksel, and M. A. Kutay, "Linear algebraic theory of partial coherence: Discrete fields and measures of partial coherence," J. Opt. Soc. Amer. A, vol. 19, pp. 1563-1571, Aug. 2002.

[54] J.-J. Xiao and Z.-Q. Luo, "Decentralized estimation in an inhomogeneous sensing environment," IEEE Trans. Inf. Theory, vol. 51, pp. 3564-3575, Oct. 2005.

[55] A. Ribeiro and G. Giannakis, "Bandwidth-constrained distributed estimation for wireless sensor networks-Part I: Gaussian case," IEEE Trans. Signal Process., vol. 54, no. 3, pp. 1131-1143, Mar. 2006.

[56] S. Nordebo and M. Gustafsson, "Statistical signal analysis for the inverse source problem of electromagnetics," IEEE Trans. Signal Process., vol. 54, no. 6, pp. 2357-2361, Jun. 2006.

[57] S. Marano, V. Matta, and P. Willett, "Quantizer precision for distributed estimation in a large sensor network," IEEE Trans. Signal Process., vol. 54, no. 10, pp. 4073-4078, Oct. 2006.

[58] J. Li and G. AlRegib, "Rate-constrained distributed estimation in wireless sensor networks," IEEE Trans. Signal Process., vol. 55, no. 5, pp. 1634-1643, May 2007.

[59] T. Oliphant, "On parameter estimates of the lossy wave equation," IEEE Trans. Signal Process., vol. 56, no. 1, pp. 49-60, Jan. 2008.

[60] T. Aysal and K. Barner, "Constrained decentralized estimation over noisy channels for sensor networks," IEEE Trans. Signal Process., vol. 56, no. 4, pp. 1398-1410, Apr. 2008.

[61] Y. Wang, P. Ishwar, and V. Saligrama, "One-bit distributed sensing and coding for field estimation in sensor networks," IEEE Trans. Signal Process., vol. 56, no. 9, pp. 4433-4445, Sep. 2008.

[62] Z. Quan, W. Kaiser, and A. Sayed, "Innovations diffusion: A spatial sampling scheme for distributed estimation and detection," IEEE Trans. Signal Process., vol. 57, no. 2, pp. 738-751, Feb. 2009.
[63] S. Joshi and S. Boyd, "Sensor selection via convex optimization," IEEE Trans. Signal Process., vol. 57, no. 2, pp. 451-462, Feb. 2009.

[64] M. Lazaro, M. Sanchez-Fernandez, and A. Artes-Rodriguez, "Optimal sensor selection in binary heterogeneous sensor networks," IEEE Trans. Signal Process., vol. 57, no. 4, pp. 1577-1587, Apr. 2009.

[65] V. Cevher and L. M. Kaplan, "Acoustic sensor network design for position estimation," ACM Trans. Sensor Netw., vol. 5, pp. 1-28, May 2009.

[66] H. Zhang, J. Moura, and B. Krogh, "Dynamic field estimation using wireless sensor networks: Tradeoffs between estimation error and communication cost," IEEE Trans. Signal Process., vol. 57, no. 6, pp. 2383-2395, Jun. 2009.

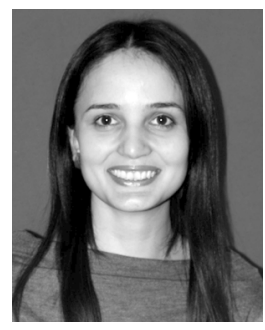

Ayça Özçelikkale (S'01-M'10) received the B.S. degree from Middle East Technical University, Ankara, Turkey, in 2004 and the M.S. degree from Bilkent University, Ankara, Turkey in 2006, both in electrical engineering.

She is currently working towards the Ph.D. degree in Bilkent University, Ankara, Turkey. Her research interests are in the area of signal processing.

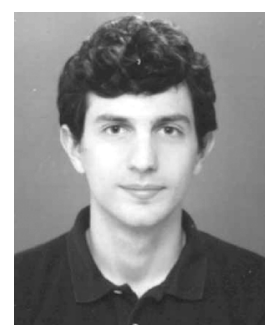

Haldun M. Ozaktas (M'07) received the B.S. degree from Middle East Technical University, Ankara, Turkey, in 1987 and the Ph.D. degree from Stanford University, Stanford, CA, in 1991.

He joined Bilkent University, Ankara, Turkey, in 1991, where he is presently a Professor of electrical engineering. In 1992, he was at the University of Erlangen-Nurnberg, Bavaria as an Alexander von Humboldt Foundation Postdoctoral Fellow. During summer 1994, he worked as a Consultant at Bell Laboratories, Holmdel, NJ. He is the author of over 90 refereed journal articles, over ten book chapters, and over 100 conference presentations and papers, over 35 of which have been invited. He is also author of the book The Fractional Fourier Transform (Wiley, 2001) and edited the book Three-Dimensional Television (Springer, 2008). His academic interests include signal and image processing, optical information processing, and optoelectronic and optically interconnected computing systems.

Dr. Ozaktas has a total of over 3000 citations to his work recorded in the Science Citation Index (ISI). He is the recipient of the 1998 ICO International Prize in Optics and one of the youngest recipients ever of the Scientific and Technical Research Council of Turkey (TUBITAK) Science Award (1999), among other awards and prizes. He is also one of the youngest members of the Turkish Academy of Sciences and a Fellow of the Optical Society of America (OSA). $\mathrm{He}$ has served as an Associate Editor of the IEEE TRANSACTIONS ON SIGNAL PROCESSING.

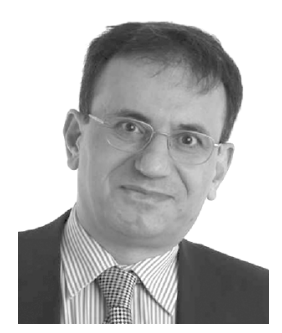

Erdal Arikan (S'84-M'86-SM'94) was born in Ankara, Turkey, in 1958. He received the B.S. degree from the California Institute of Technology, Pasadena, CA, in 1981, and the S.M. and $\mathrm{Ph} . \mathrm{D}$. degrees from the Massachusetts Institute of Technology, Cambridge, MA, in 1982 and 1985, respectively, all in electrical engineering.

Since 1987, he has been with the Electrical-Electronics Engineering Department of Bilkent University, Ankara, Turkey, where is currently a Professor. His research interests are in information theory and 